\title{
Faire la police dans la Manche : L'évolution de la coopération transfrontalière (1968-1996)
}

James Sheptycki

\section{OpenEdition}

\section{Journals}

Édition électronique

URL : http://journals.openedition.org/conflits/367

DOI : $10.4000 /$ conflits.367

ISSN : $1777-5345$

Éditeur :

CCLS - Centre d'études sur les conflits lilberté et sécurité, L'Harmattan

Édition imprimée

Date de publication : 15 octobre 1997

ISSN : 1157-996X

Référence électronique

James Sheptycki, «Faire la police dans la Manche : L'évolution de la coopération transfrontalière (1968-1996) », Cultures \& Conflits [En ligne], 26-27 | automne 1997, mis en ligne le 15 mars 2006, consulté le 30 mars 2021. URL : http://journals.openedition.org/conflits/367 ; DOI : https://doi.org/ $10.4000 /$ conflits.367

Ce document a été généré automatiquement le 30 mars 2021

Creative Commons License 


\title{
Faire la police dans la Manche : L'évolution de la coopération transfrontalière (1968-1996)
}

\author{
James Sheptycki
}

\section{James SHEPTYCKI ${ }^{1}$}

\section{Introduction}

Depuis les années 1990, criminologues et sociologues s'intéressent à l'européanisation de la police. Cela tient au fait que ce problème est devenu politique, surtout depuis la signature du traité de l'Union européenne. Or, bien avant cette date, les policiers ont mis en oeuvre l'internationalisation des missions de police, sans que personne ne se soit vraiment penché sur le travail accompli par ces précurseurs. Cet article essaie de retracer cette évolution de la transnationalisation de la police en Europe de l'Ouest au cours de ces vingt-cinq dernières années. Pour ce faire, nous avons utilisé des archives conservées en Angleterre, en Belgique, en France et aux Pays-Bas et, en particulier, les archives de la Cross Channel Intelligence Conference (CCIC), un organisme établi dès 1968 pour mettre en place la police transnationale dans la Manche. Ces documents ont permis une reconstruction sociologique du processus d'organisation qui a abouti à la transnationalisation de la police dans ce secteur. Outre ces documents, 471 dossiers d'opérations provenant de l'European Liaison Unit (ELU), l'unité européenne de liaison, basée à Folkstone dans le Kent, ont été consultés. À ces éléments d'archives, il faut ajouter de nombreux entretiens avec des policiers en poste et des notes de terrain des policiers ; cela nous a permis d'étudier les réseaux personnels interpoliciers qui ont bâti l'infrastructure policière actuelle ${ }^{2}$. Les quatre phases du développement de la police transnationale on peut diviser cette genèse du transnational en quatre périodes. La première phase, qui va de 1968 à 1970, est celle de la fondation du réseau policier transnational et de l'établissement de ses pratiques de travail. La seconde, qui est la plus longue dans l'histoire de la coopération policière dans la Manche, va de 1971 à 1985. Au cours de cette période, le travail de la CCIC et les rapports personnels entre les officiers de liaison furent le moteur de l'échange d'informations entre les polices des 
frontières concernées. Interpol intervint aussi, mais comme nous le verrons plus loin, le réseau local fut beaucoup plus efficace. La troisième phase va de 1986 à 1991. Elle présente les caractéristiques d'une phase intermédiaire, d'un " interrègne ", au cours de laquelle le projet de tunnel sous la Manche était en cours. La police en Europe retint dès lors l'attention des gouvernements nationaux et la structure de la police transeuropéenne devint très élaborée, tant verticalement qu'horizontalement. Pendant cette période, la CCIC, qui était un forum d'échange d'informations, se transforma en une table ronde sur la politique policière. Les questions de l'harmonisation des lois et de l'organisation de la transnationalisation furent au centre des débats, surtout après 1986. À partir de là, l'échange d'informations fut bouleversé par la révolution de la communication et, en 1991, la police transfrontalière entra dans sa phase actuelle. L'importance de la CCIC en tant que forum d'information diminua. De nos jours, la création de l'Unité européenne de liaison, basée à Folkstone, au terminal du tunnel sous la Manche, et l'établissement du projet Linguanet ont modifié la donne. La police transnationale n'est plus un fait d'exception mais une activité entrée dans les moeurs, une activité routinière dans cette région d'Europe. La fondation L'un des premiers fondateurs de la CCIC fut le chef de la police du Kent, Sir Dawney Lemon (CBE). Lors de la première rencontre de la CCIC à Maidstone, dans le Kent, il déclara qu'il souhaitait voir se développer des relations personnelles entre policiers anglais, belges et français en poste dans la zone frontalière de la Manche. Il affirma : " Les côtes du Kent s'étirent sur plus de 120 miles. Il est impossible de surveiller une telle étendue, mais il est possible d'accumuler les renseignements sur les personnes ayant des activités criminelles dans le Sud-Est de l'Angleterre et d'agir contre elles. Ceci ne peut se faire qu'en renforçant les agences de police, engagées des deux côtés de la Manche, en travaillant ensemble, en rassemblant nos renseignements afin de battre notre ennemi commun : le criminel "3. Lemon évoqua la nécessité d'une meilleure compréhension des lois et des procédures nationales afin que les problèmes des uns et des autres fussent mieux appréhendés; il espérait qu'il en sortirait une " coopération plus étroite ". Les réunions finales de cette conférence et de la suivante, qui se déroula à Bruges six mois plus tard, furent donc consacrées aux principes juridiques et organisationnels qui régissaient les forces de police impliquées. Replacée dans le contexte d'une politique européenne moins étroite, il convient de souligner que cette recherche d'une alliance policière infra-régionale, précède l'entrée du Royaume-Uni dans la CEE. Une entrée à laquelle s'était déjà opposé, le 14 janvier 1963, le général de Gaulle qui, en 1966, retira la France de l'OTAN. L'initiative de la CCIC démontre simplement la difficulté, voire l'impossibilité, de découpler les États européens les uns des autres. En 1973, quand le Royaume-Uni entra formellement dans la Communauté économique européenne, la transnationalisation de la police des frontières de la Manche était déjà institutionnalisée depuis presque cinq ans. Un travail policier qui ne devait intéresser le monde politique que beaucoup plus tard. Même si la transnationalisation de la police n'était pas la préoccupation majeure des hommes politiques, certains des problèmes abordés par les policiers les intéressaient au premier chef. Il suffit de se rappeler le discours prononcé le 9 avril 1966 par le député Enoch Powell sur " les rivières de sang " pour le deviner. Les minutes de la première conférence de la CCIC tenue à Maidstone montrent qu'un thème précis retenait déjà l'attention de l'institution : les immigrants clandestins. Les dossiers contenaient 22 cas de trafic de main d'oeuvre immigrée. Ils étaient suffisamment révélateurs pour permettre une caractérisation générale du modus operandi de cette forme de criminalité. À cette époque, les immigrants 
clandestins que la police britannique cherchait à refouler venaient pour la plupart du Pakistan, de l'Inde et de l'Iran. Il y eut aussi des cas de marins, venus de tout le Commonwealth, quittant leurs navires dans les ports anglais. Une des routes parmi les plus empruntées par les clandestins pakistanais passait par l'Iran, la Turquie, la Grèce, la Yougoslavie, l'Autriche, l'Allemagne de l'Ouest et se terminait aux " avant-postes " en France et en Belgique, avant le passage final en Angleterre. D'après nos sources, le prix par personne d'un passage revenait aux environs de $600 £$. Les réseaux de passeurs étaient multinationaux, les chaînes incluant de nombreuses nationalités, dont des SudAfricains, des Mauriciens, des Belges, des Français, des Néerlandais et des sujets britanniques. À l'évidence, l'immigration clandestine nécessitait une réponse internationale de la part de la police. Les minutes des conférences de la CCIC entre 1968 et 1970 signalent également des affaires de drogues : haschich, marijuana et opium. Des trafics organisés disait-on par des Nord-Africains, des Turcs, les " beatniks " de Londres et leurs homologues des Pays-Bas. Des affaires qui prendront plus tard une ampleur beaucoup plus grande, comme nous allons le voir. La police dans la région de la Manche avait également affaire à d'autres activités criminelles, en particulier le transport de biens volés. Les activités des antiquaires, dont on pensait qu'ils pouvaient être impliqués dans le recel et le trafic d'objets d'art et d'antiquités, étaient surveillées. Le transport de véhicules volés était également un problème majeur. À la fin des années 1960, la police de la Manche était confrontée à une hausse du crime transfrontalier, et des tentatives étaient faites pour mettre en place une coopération policière et $\mathrm{y}$ répondre. Néanmoins, si tout le monde acquiesçait au fait qu'une coopération policière devait être mise en place, la question se posait de savoir quels pays et quelles institutions policières allaient y participer. Les institutions policières dans la coopération transfrontalière La CCIC tint deux conférences en 1970 à une semaine d'intervalle. La première eut lieu à Brighton et, à l'exception du commissaire principal de la police de Bruges, tous les participants étaient Britanniques. En tout, huit services de la police britannique étaient représentés, en plus de la Police métropolitaine de Londres (Special branch) : il s'agissait des représentants du Devon, de la Cornouaille, des villes de Dorset et Bournemouth, de la police des comtés de Sussex, Surrey, Hampshire, et de celles du Kent et de l'Essex. Le propos de ce meeting des officiers de police était de consolider le réseau tissé entre les officiers de liaison anglais de tout le Sud de l'Angleterre. Les minutes de la réunion indiquent que les policiers des huit forces de police du Sud et de la Metropolitan Police négocièrent afin d'établir " que les accords entre les diverses forces de police, celle des comtés et la police métropolitaine, étaient suffisamment adéquats et fonctionnels, notamment en ce qui concernait l'échange de renseignements ". Ces hommes étaient, pour la plupart, des officiers de liaison désignés par leurs corps respectifs ${ }^{4}$. À la seconde réunion, celle de Bruges, un nouvel acteur de ce partenariat transnational intervint : le commissaire principal de la police judiciaire de Bruges, Jacques Matthys, qui avait été l'un des fondateurs de la CCIC. Dans son discours d'ouverture, il déclara : " il y avait encore une place vide dans notre cercle, aujourd'hui je présume que nous avons comblé ce vide. Nous avons le grand plaisir d'accueillir les représentants officiels de la police du royaume des PaysBas "5. Désormais, tous les États frontaliers de la Manche étaient formellement réunis au sein d'une entreprise policière frontalière commune. Il faudrait à ce stade définir les organismes membres de ce réseau. Les organismes policiers participants La police britannique, rappelons-le, est organisée territorialement. Chaque force de police est responsable d'une zone géographique délimitée. La police est en théorie contrôlée 
grâce à un accord tripartite entre le gouvernement central, le gouvernement local et les commissaires de police. Les Chiefs Constables $^{6}$ bénéficient d'une relative indépendance grâce à la doctrine de la Constabulary independence et à la notion de décisions opérationnelles de police, ce qui explique en partie la facilité avec laquelle la CCIC put se développer du côté britannique. Tous les officiers de police se virent (en principe) attribués les pouvoirs de police sur l'ensemble du territoire du Royaume-Uni par le Police Act de 1964, mais la Special Branch fut le seul corps de police qui eut une entière compétence au niveau national, avant la création en 1992 du National Criminal Intelligence Service (NCIS) ${ }^{7}$. D'autres organismes représentatifs au niveau national participèrent occasionnellement aux réunions de la CCIC, y compris des hommes appartenant au Her Majesty's Inspectorate of Constabulary (HMIC), au Home Office ${ }^{8}$ (ministère de l'Intérieur), et à un corps intermédiaire, incluant la police des frontières, les Regional Crime Squads, qui facilite les enquêtes de police. À l'occasion, des officiers du South East Regional Crime Squad participèrent également aux conférences. D'une manière générale, les officiers de liaison des huit institutions policières locales mentionnées ci-dessus, formaient l'essentiel de la police anglaise présente dans le secteur de la Manche. Ils étaient, bien sûr, en contact plus ou moins régulier avec des officiers des Douanes (HMCE) et des services de l'Immigration (HMIS), mais il apparaît clairement que leur rôle était plus limité que celui de leurs collègues policiers. Quoiqu'il y eût des frictions administratives entre ces trois services, la police avait historiquement une sous-culture spécifique relativement homogène. Cela tient à l'entraînement dispensé au Collège de Police de Bramshill qui accueille les officiers junior et senior. Enfin, il convient de souligner que, contrairement à leurs collègues du continent, les policiers en Angleterre et au Pays de Galles ont la responsabilité de la conduite des enquêtes criminelles et qu'ils ne prennent que rarement conseil auprès des autorités judiciaires. En conséquence, les représentants d'institutions, comme le directeur des enquêtes judiciaires, n'ont participé qu'accidentellement aux réunions de la CCIC. Les polices continentales Les Pays-Bas Les forces de police s'articulant différemment sur le continent, c'est le système néerlandais qui se rapproche peut-être le plus du système britannique. Dans les années 1960, il existait 148 services de police municipale, et une Police d'État, la Rijkspolitie, couvrant les zones urbaines et rurales qui n'étaient pas du ressort des polices municipales. Cette dispersion des unités de police les rendait difficiles à diriger, gênait les transferts de ressources et d'informations et a entraîné une vaste réforme des institutions policières ; une réforme devenue nécessaire pour répondre aux besoins de coordination au niveau national et de coopération internationale. Ce fut la loi de Police de 1993 qui fusionna en un corps unique la police municipale et la Rijkspolitie, service divisé en 25 unités régionales et une unité nationale fournissant les services, incluant le CRI (équivalent néerlandais du $\mathrm{NCIS})^{9}$. Les Pays-Bas furent représentés à la CCIC le plus souvent par des officiers de la police de Rotterdam et parfois par des officiers du Bureau National d'Interpol de La Haye (NCB). Des études ont montré que les relations entre ces forces de police pouvaient fonctionner avec succès à travers ces réseaux informels ou souterrains et non par les moyens administratifs formels à disposition. Avant 1993, on se trouvait donc en présence d'un " patchwork " d'unités de police autonomes. L'inconvénient d'une telle situation résidait dans la difficulté de gérer et de transférer les ressources, notamment l'information ${ }^{10}$. La police belge Le système belge est très différent. Les policiers qui représentèrent la Belgique appartenaient à la police judiciaire. Celle-ci, qui à l'époque comprenait 22 brigades locales, est responsable formellement devant le juge 
d'instruction. Il convient de signaler que, contrairement aux juges néerlandais ${ }^{11}$, qui ont les mêmes attributions qu'eux, des juges belges participèrent aux réunions de la CCIC. Fondée en 1919, la police judiciaire belge, qui regroupe environ 1400 officiers, n'a eu de compétence nationale qu'en 1964. C'est aujourd'hui un amalgame complexe d'institutions diverses, chargé d'enquêter sur le crime, et particulièrement sur le crime organisé, le terrorisme, le trafic de stupéfiants et le crime transfrontière. Encore aujourd'hui, elle n'a pas de juridiction administrative unifiée; c'est au contraire une mosaïque de textes et de règles juridiques qui la régissent. Le recrutement s'y est longtemps fait sur une base locale, avec une formation empirique sur le terrain au cours d'une année de probation, sans aucun programme d'études unifié, jusqu'à la création en 1984 (déjà proposée en 1971 mais retardée) de l'Institut des Hautes Études policières, destiné à harmoniser l'entraînement des policiers. Un tel cursus est devenu obligatoire en 1989. La nature fragmentée de cette structure organisationnelle ${ }^{12}$ est en partie contrebalancée par le Commissariat général qui, outre la coordination de la documentation au niveau national, accueille le bureau central national belge d'Interpo ${ }^{13}$ et coordonne la coopération policière internationale à tous les niveaux. La Gendarmerie belge ne participa pas aux réunions de la $\mathrm{CCIC}^{14}$. Le système français Le système policier y est un peu moins compliqué que celui de la Belgique. Il y a dans la littérature de fréquentes références à un " modèle français "15, qui insistent sur son degré de centralisation. En fait, ce système se présente comme une agrégation institutionnelle hautement différenciée. Ce niveau de différenciation peut s'expliquer par l'histoire de l'État français ${ }^{16}$. Trois organisations principales forment ce système : la Police nationale, la Gendarmerie et la Police municipale. Les deux premières sont des forces nationales très centralisées, l'une relevant du ministère de l'Intérieur, l'autre du ministère de la Défense. Toutes les deux mènent des enquêtes criminelles et ont affaire au ministère de la Justice. La fondation de la Gendarmerie remonte bien avant la Révolution; quant à la police nationale, elle est le fruit de la fusion, en 1966, entre la vieille Sûreté et la Préfecture de Police de Paris. Il est essentiel ici de rappeler que les systèmes policiers français sont le produit d'une histoire longue, ponctuée de mouvements sociaux et de changements de Constitution. Le résultat, paradoxal, aura été la création d'un système de police très centralisé, avec des conflits et des rivalités d'institution, comme la " guerre des polices " entre la gendarmerie et la police nationale l'a montré. Mais ces conflits peuvent être internes et concerner un corps, comme la police nationale ${ }^{17}$. Jusqu'en 1945, dans la majorité des villes la police était organisée localement ; c'est après la Seconde Guerre mondiale que le gouvernement a cherché à asseoir son contrôle à l'échelon local, ce qui a déclenché des contestations très fortes, comme à Lille ${ }^{18}$. En réaction à la centralisation, la volonté de contrôle local a resurgi avec force à la fin des années 1980. Les archives de la CCIC indiquent, comme dans le cas de la Belgique, que la Gendarmerie n'était pas active dans la coopération internationale policière. En revanche, trois départements de la police nationale sont très actifs. Il s'agit de la Police judiciaire, de la Police de l'air et des frontières (PAF) et des Renseignements généraux. La première pratique une police d'investigation sous le contrôle d'un magistrat instructeur. La Police de l'air et des frontières, qui est devenue un corps séparé de la Police judiciaire en 1969, dépend du ministère de l'Intérieur (via son directeur général) et contrôle les mouvements des personnes aux frontières ( $\mathrm{y}$ compris les aéroports). Ces deux institutions furent les plus actives. Ont été également représentés les Renseignements généraux et les membres du Bureau Central d'Interpol de Saint-Cloud. Nous avons inclus Interpol dans la catégorie des représentants officiels 
français car il était, par essence, une extension du ministère de l'Intérieur, qui supporta la majeure partie du fardeau financier et a offert à Interpol quatre secrétaires généraux entre 1946 et $1986^{19}$. La Police nationale est très segmentée, verticalement et horizontalement, ce qui conduit à des frictions administratives. Comme dans le cas belge, ceci a donné lieu à des difficultés dans la direction et le transfert des ressources, notamment de l'information. Néanmoins, à l'inverse de la Belgique, il existe depuis longtemps un système d'entraînement unifié. Il est fonction du niveau auquel opérera la recrue, mais il est significatif de constater que tous les officiers de police passent par l'École nationale de Police de Saint-Cyr-au-Mont d'Or, ce qui assure dans les rangs de l'encadrement une homogénéisation de la culture institutionnelle. Si ce système peut poser de graves problèmes de coordination interne, en termes de coopération policière transfrontalière, le problème paraît être celui de la centralisation de l'information dispersée, qui crée des chaînes de communication conduisant à l'inefficacité. Ce fait est en partie atténué par le système des Services régionaux de Police judiciaire, qui a pour tâche de coordonner des enquêtes sur une large échelle et est analogue au système régional britannique des Crime Squads. L'insistance sur le cheminement des informations à travers une administration centralisée appert des minutes de la CCIC : lors de la conférence du 16 avril 1969, tenue à Lille ${ }^{20}$, le chef du service régional de Police judiciaire notait que les relations entre les ports étaient suffisamment bonnes pour autoriser un contact direct transfrontalier entre officiers de police en cas d'affaire urgente, et recommandait que " l'information pour tous les ports français de la Manche soit envoyée sous la forme de messages au bureau central national ". Un an plus tard, le Chief Constable adjoint du Sussex demanda que l'on désigne des hommes de liaison dans toutes les unités de police qui participaient à la CCIC, " afin d'éviter l'intervention directe des chefs "21. La réponse belge fut de désigner quatre officiers de liaison en fonction des types d'infractions (généralités, vol, trafic de stupéfiants, faux et immigration). La réponse du commissaire de police chargé $\mathrm{du} B C N$ d'Interpol à Paris fut négative : " Il était impossible pour la France de désigner un contact par niveau local ". À la conférence de l'année suivante, la question des communications directes à un niveau opérationnel entre policiers refit surface dans un contexte de discussions sur le contrôle des croisières de plaisance dans les eaux côtières et dans la Manche, ainsi que sur l'importation de véhicules volés ${ }^{22}$. Il ressortit de ces échanges que nombre d'informations envoyées en France n'étaient pas transmises aux services concernés et que, de plus, la Belgique et les Pays-Bas ne recevaient pas d'informations des policiers français. On réaffirma le principe de la communication directe des informations mineures entre les parties intéressées; Interpol fut reconnu comme le réseau de communication approprié des informations de nature officielle, entre les forces de police. On pensait qu'il fallait travailler encore plus à la création de solides contacts avec les responsables policiers locaux de l'Hexagone. Il ressort de ces discussions que la police transnationale est le résultat d'un mélange institutionnel complexe, difficile à orchestrer. Néanmoins, la culture policière, indépendamment de ses particularismes nationaux, implique un très fort sens de la mission. La perception par les policiers du poids du crime transfrontalier conduisit à accroitre l'effort pour construire ce réseau. Compte tenu de la complexité organisationnelle d'une telle initiative, la question se pose naturellement: comment aujourd'hui, ce système de police transnationale fonctionne-t-il ? Il est clair que les policiers membres de la CCIC forment le noyau de cette police transnationale de la Manche ; il faut maintenant démonter les mécanismes fondamentaux de la police transnationale et évaluer son fonctionnement actuel. La 
police transnationale à l'âge du télex Au cours des deux phases que nous avons identifiées, le fonctionnement de la CCIC fut celui d'un forum d'échange d'informations sur les criminels connus opérant dans la région de la Manche. On rendait compte d'opérations réussies, mais il était plus important de faire circuler les informations sur les criminels recherchés. Les représentants d'Interpol, tout comme les polices française, belge, néerlandaise et britannique, saisirent l'occasion de faire circuler leurs avis de recherche sur les personnes dont on soupçonnait qu'elles pouvaient être dans la région. La circulation se faisait normalement en fonction du type de délit et, comme nous l'avons déjà mentionné, dès le début, la question de l'immigration fut prioritaire. Mais, quand les Pays-Bas organisèrent leur première conférence à Rotterdam en 1973, cette question fut partiellement éclipsée par le trafic international de stupéfiants. On y évoqua également d'autres types de délits, les fraudes, les vols, les vols avec voies de fait. Photographies, empreintes digitales, avis de recherches étaient facilement mis à disposition au cours de ces réunions. Le second mode de fonctionnement de la transnationalisation policière découle du rôle de la CCIC et concerne les officiers de liaison. On peut citer un premier exemple, celui d'un sergent détective de la police du Kent, qui reçut de ses supérieurs l'autorisation de se rendre à Calais, Dunkerque et Boulogne, pour rassembler des informations ${ }^{23}$. De son côté, la police belge avait désigné trois officiers de liaison pour faciliter la collecte des informations et on décida de demander à la direction centrale de la police judiciaire française d'autoriser la mise en place d'un point de contact similaire à Lille. Le fonctionnement de ce type d'accords a mis en lumière nombre de problèmes pratiques, liés au schéma fonctionnel complexe que nous venons de décrire. Une question centrale fut le degré d'investissement d'Interpol vis-à-vis de ce qui était qualifié de renseignement " de basse-intensité ". Un cas de figure évoqué fut celui de criminels identifiés lors du passage des postesfrontière. De même, que fallait-il faire des noms, des adresses, des numéros de téléphone trouvés en possession d'un délinquant arrêté à l'étranger? Comment notifier au pays d'origine l'arrestation d'une personne? Dans tous ces cas, on ne voyait pas clairement quelle autorité policière serait chargée de la transmission des informations. En même temps, il était clair que le canal d'Interpol n'était pas le moyen idéal ; il ne pouvait avoir la réactivité requise par des officiers de police en opération, en raison du volume potentiel de ces informations qui risquait de submerger le système très centralisé et donc très long d'Interpol ${ }^{24}$. En 1973, la conférence de Rotterdam permit d'aplanir les difficultés en définissant trois lignes de conduite ${ }^{25}$. Tout d'abord, il fut décidé que les renseignements urgents et opérationnels seraient communiqués directement aux policiers membres de la CCIC, mais ces informations ne seraient fournies aux autres forces de police qu'à partir d'une structure officielle internationale : Interpol. Il y avait donc une communication de force à force, il était de la responsabilité de chaque police d'alerter le bureau central national d'Interpol. Il fut aussi reconnu en cette occasion que le renseignement criminel soulignait la nécessité de mener des enquêtes transfrontalières prolongées dans le temps. Lors de ces enquêtes, il fut décidé d'organiser des réunions multijuridictionnelles au niveau tant stratégique qu'opérationnel. Finalement, Interpol fut reconnu comme le seul moyen approprié de circulation des informations, telles que les photographies, les empreintes, les fichiers de délinquants, etc. Cinq ans plus tard, les discussions amenèrent à faire un bilan des relations entre les services ${ }^{26}$. " ...Le Secrétaire Général d'Interpol considérait que les objectifs de la Conférence étaient clairement définis. Interpol acceptait sans réserves le principe des contacts frontaliers, qui, cependant, devaient s'opérer en 
harmonie avec le schéma général de la coopération organisée par Interpol... " mais, disait-il, " il est évident que la participation de représentants du Secrétariat général international et des bureaux nationaux concernés... ne pouvait être qu'avantageuse pour toutes les parties ". La majeure partie du réseau de communication de police à police dans le secteur de la Manche était alors en place: il y avait un télex reliant Bruges et le Kent ainsi que des contacts téléphoniques; pourtant, l'essentiel, à savoir les communications détaillées, se faisait toujours lors des réunions, c'est à dire d'homme à homme. Pour illustrer ce principe de fonctionnement, examinons en détail une opération réussie. Un cas pratique de police transnationale Lors d'une conférence de la $\mathrm{CCIC}^{27}$, un groupe de trafiquants de drogue fut identifié. C'était le début d'une enquête transnationale qui devait durer neuf ans avant de porter complètement ses fruits et d'englober la moitié de la planète. Au départ, les perspectives étaient moins grandioses. Les dossiers comprenant les noms et les fonctions détaillées des personnes connues comme étant des passeurs et des hommes de paille circulèrent. Il fut révélé que l'un des passeurs lié au groupe avait été arrêté à Douvres en juillet 1976 en possession de $40 \mathrm{~kg}$ de cannabis libanais. Son interrogatoire permit la découverte de ramifications dans le Sud des Pays-Bas et d'une boîte aux lettres à Bruxelles. On soupçonnait également l'implication d'individus à Amsterdam. Les responsables des brigades des stupéfiants londoniens, du Kent et de l'Essex, avec les officiers des douanes, venaient de remporter ce que l'on peut qualifier de succès limité. Mais pour le parfaire, on décida de continuer l'enquête en faisant appel aux autres services policiers nationaux afin d'enquêter sur toutes les activités de ce réseau. Un an plus tard, l'arrestation de 70 individus en possession de petites quantités d'amphétamines achetées aux Pays-Bas fut signalée ${ }^{28}$. Ces arrestations menèrent à la localisation d'un laboratoire d'amphétamines fonctionnant à la frontière de la Belgique et des Pays-Bas. En août 1977, lors de la session de Maidstone, on apprit qu'une presse à fabriquer des comprimés avait été découverte. La personne arrêtée avait cédé quelque $10 \mathrm{~kg}$ de poudre d'amphétamine néerlandaise, afin de fabriquer des pilules à destination du marché britannique. Les occupants de la maison étaient aussi impliqués dans le transport de $150 \mathrm{~kg}$ de résine de cannabis, qui avaient été introduits dans des chauffeeau qu'importait une entreprise d'import-export établie aux Pays-Bas. Il était désormais établi que le réseau s'étendait sur trois pays et fournissait des amphétamines et du cannabis. Des mandats d'arrêt pour trafic de drogues, concernant quatre individus, furent fournis un an plus tard, lors de la conférence de Chelsmford ${ }^{29}$. Avant même ce stade, l'enquête transnationale avait été suffisamment élaborée et formalisée pour être baptisée Opération Lager. Les minutes de la conférence indiquent que l'enquête avait nécessité de longues périodes de surveillance dans les trois pays et le contrôle des frontières, y compris internationales, par les officiers des douanes. Les conférences suivantes ${ }^{30}$ indiquent que des moyens encore plus intrusifs avaient été utilisés : écoutes téléphoniques, contrôle des télex et des transactions bancaires et visite des locaux du groupe, où de la correspondance écrite et d'autres indices avaient été collectés. Il semble que la police belge arrêta sur son territoire uniquement ses nationaux, et que la police britannique fit de même, arrêtant et inculpant plus de 100 personnes. En revanche, la police néerlandaise arrêta des ressortissants britanniques liés au réseau, comme ces deux Britanniques qui avaient placé $254 \mathrm{~kg}$ de cannabis dans un faux réservoir de camion. Les sources britanniques indiquèrent que ce véhicule avait fait huit allers et retours entre le continent et le Royaume-Uni. On put prouver ensuite qu'une vingtaine de ce type de voyages avaient été faits par ce seul véhicule! 
L'Opération Lager fut momentanément close en 1981. Plusieurs des membres importants du réseau avaient certes été identifiés, mais ils avaient échappé aux policiers et, faute d'information, on ne pouvait aller plus loin. L'opération reprit inopinément en 1982, grâce à l'arrestation de l'un de ces individus à Amsterdam, pour une autre affaire. Son extradition vers le Royaume-Uni fut difficile, mettant en évidence les barrières juridiques liées aux jurisprudences des deux pays. Son extradition devait se faire au motif d'association de malfaiteurs en vue d'importer de la drogue en Angleterre. On s'aperçut tardivement que la loi néerlandaise ne reconnaissait pas comme suffisant le motif d'association de malfaiteurs (conspiracy). Il fallut reconstituer un dossier montrant que le trafic était bien le fait de cette personne. Un officier britannique dut se déplacer spécialement et remettre de la main à la main aux autorités néerlandaises le nouveau dossier, un jour avant l'extinction légale de la procédure aux Pays-Bas. Ceci ne mit pas fin à la coopération entre les deux polices. Une fois le trafiquant extradé (malgré des interjections en appel à toutes les étapes, auprès des Cours criminelles néerlandaises), la police néerlandaise, à la demande de ses collègues britanniques, rassembla les témoins et les convoya en Angleterre afin qu'ils puissent apporter leur témoignage. Fin février 1984, une lettre du bureau d'Interpol de New Delhi, datée du 17 janvier, arriva à Londres, ouvrant le chapitre final de l'Opération Lager. Elle signalait à Interpol l'arrestation à Bombay de deux ressortissants britanniques en possession de $1746 \mathrm{~kg}$ de cannabis qu'ils s'apprêtaient à exporter; étaient jointes les photos des deux prévenus, dont l'un avait été identifié comme l'un des principaux organisateurs du réseau traqué par les policiers de l'Opération Lager. De plus, la saisie de nombreux passeports permit de retracer les activités du réseau en Afrique, dans le Sud de l'Europe et en Inde. Le principal intéressé parvint à s'échapper de prison et quitta l'Inde avec un faux passeport. Fortuitement, la surveillance prolongée d'un individu lié à ce réseau, mais soupçonné de trafic d'oeuvres d'art volées, permit la conclusion finale de l'enquête en août 1985. L'homme-clé fut arrêté, un trafic international de stupéfiants se chiffrant en millions de dollars était enfin démantelé. Il résulte de cette série d'enquêtes que la libre circulation des informations a fonctionné entre tous les policiers impliqués dans l'opération. Une fois lancée hors du RoyaumeUni, l'opération et sa réussite auront été fonction de la coordination légale de l'action des polices des différents pays. À plusieurs reprises, le Directeur des Enquêtes publiques et les autorités judiciaires délivrèrent des Commissions rogatoires. Au cours de ces enquêtes, nombre de policiers britanniques se rendirent aux Pays-Bas, en Belgique, en France et en Espagne (l'un d'entre eux huit fois). Plusieurs réunions se tinrent en Belgique, aux Pays-Bas et au Royaume-Uni, et impliquèrent, en plus des policiers, les autorités judiciaires. Ces conférences eurent deux fonctions : d'abord, permettre la circulation de l'information et, ensuite, négocier en fonction de la jurisprudence, afin de faciliter les arrestations et les poursuites judiciaires. Les informations et les enquêtes périphériques furent aussi incorporées pour la bonne marche de l'Opération Lager. Cette opération mit en évidence nombre de barrières, liées à la souveraineté, qui gênaient les opérations de police transnationale. Une enquête sur des ressortissants néerlandais dont on soupçonnait les liens avec des réseaux opérant dans le Sud de la France ne put aboutir, en dépit de preuves suffisantes et d'une coopération policière étroite, en raison d'un trou dans la législation sur l'extradition en matière d'affaires de drogue entre la France et les Pays-Bas. En même temps, les archives de la CCIC révèlent qu'en 1978 une enquête sur un trafic d'héroöne en provenance d'Extrême-Orient, impliquant des agents canadiens opérant sous 
couverture, se solda par des arrestations en Belgique et en Grande-Bretagne, pays qui avaient conclu des traités d'extradition avec la France. En revanche, les individus arrêtés aux Pays-Bas furent mis sous bonne escorte à bord d'un ferry à destination de l'Angleterre où ils furent arrêtés de nouveau et extradés vers la France ${ }^{31}$. Les occasions de manoeuvre similaire au cours de l'Opération Lager furent activement recherchées, sans que les archives de la CCIC postérieures indiquent s'il y eut d'autres développements de cet aspect de la coopération policière. Au regard des possibilités de tourner les barrières juridiques, la souveraineté gêna les enquêtes dans d'autres domaines. Les enquêtes menées en Espagne " furent les plus compliquées du point de vue de la jurisprudence " ${ }^{32}$. Le fait qu'aucune arrestation n'ait eu lieu sur le territoire espagnol et que rien ne prouve que la juridiction espagnole ait été utilisée en vue de poursuites (ce qui, d'un autre point de vue, a sans doute aidé la conduite de l'enquête elle-même) suggère que ces barrières juridiques n'étaient toujours pas surmontées à la fin de l'enquête. Les enquêtes se déroulèrent en Europe (France, Espagne, Suisse, R.F.A.), aux Bahamas, en Afrique de l'Ouest et de l'Est et en Inde. Ces exemples montrent que la coopération entre les services de police fut toujours soutenue mais, en même temps, entravée par les barrières juridiques. Les officiers de police tirèrent euxmêmes les conclusions de l'Opération Lager ${ }^{33}:$ " ...Une meilleure compréhension des problèmes quotidiens rencontrés par les différents services de police a été obtenue. Il est très clair que sans les déplacements réciproques des officiers de police des divers pays, une enquête internationale est au-dessus de nos moyens... ". " Le succès d'une enquête en matière de trafic de stupéfiants demande la coopération totale des services engagés. C'est un impératif local, national et international... ". Les policiers en charge de l'Opération Lager ont bénéficié de cette " entière coopération dans tous les pays où l'enquête les a conduits ". Il est néanmoins " important de souligner que la forme de liaison la plus importante et la plus efficace fut celle conduite au niveau pratique, qui a permis l'échange d'informations ", tandis " que les exigences juridiques étaient satisfaites au cas par cas ". Ce cas de transnationalisation policière éclaire certains faits. Premièrement, une enquête de police transnationale ne peut pas fonctionner sur la seule base du renseignement: comme les arrestations effectuées le montrent, les procédures entamées pour des délits commis in situ, sans recours à l'extradition, posent problème, même si la nature du délit est transnationale. Ensuite, le travail à une échelle internationale peut être exécuté sur la base d'une communication directe de police à police sans respecter les schémas institutionnels bureaucratiques. Comme les minutes de la CCIC le prouvent, Interpol n'aura été impliqué dans la conduite de l'opération que tangentiellement. Enfin, les barrières juridiques peuvent être tournées ; néanmoins, du point de vue juridique, cette option expéditive n'est nullement l'option idéale ${ }^{34}$. Enfin, la police transnationale est une entreprise progressive, en raison de l'accumulation continuelle de succès à la marge. À cet égard, il est significatif qu'après l'Opération Lager les autorités britanniques aient décidé de détacher à La Haye un officier de liaison, afin de créer un lien permanent avec la police et les douanes néerlandaises. Ces développements sont instructifs. On imagine souvent que la police est l'expression de la souveraineté d'État et n'a de rapports que vis-à-vis de l'ordre interne des États-Nations. De ce point de vue, il est bien difficile de penser l'action policière quand elle transgresse les frontières d'État, et l'on pourrait suggérer que cette action n'est même pas envisageable dans la pratique. Pourtant, les développements apparus dans la pratique policière entre 1968 et 1986 démontrent que des relations policières transnationales, encore impensables quelques années auparavant, des 
réseaux informels de policiers engagés dans l'échange de renseignements se mettaient en place. Pour paraphraser Malcolm Anderson ${ }^{35}$, il y a quelques années, une telle initiative aurait été au mieux jugée inconvenante, au pire prise pour de l'espionnage. La transnationalisation policière et les politiques de l'Europe Après 1986, la nature des conférences de la CCIC évolua. Le débat sur les barrières juridiques, les exposés sur les différents systèmes policiers et les accords de liaison formèrent le socle de la CCIC. L'échange d'informations et les " debriefings " d'opérations réussies ne tinrent jamais ce rôle. Mais, la marche vers le marché unique européen modifia les préoccupations des policiers. Pour reprendre les termes du commissaire principal de la PJ de Bruges, Paul van Hecke, un des premiers participants aux conférences, " les polices souhaitaient la création d'une institution policière avec des pouvoirs opérationnels dans toute l'Europe de l'Ouest et une législation pratique unifiée au sein de ce nouvel espace géographique "36. Il soulignait également que si, dans un passé récent, " évoquer simplement l'extension des intérêts européens à des problèmes tels que le combat contre le terrorisme, le trafic de stupéfiants et le crime organisé, était considéré comme un coup de poignard dans le dos de la souveraineté nationale ", le temps était venu de mettre en place une politique européenne tirant les enseignements de la future mise en place du marché unique européen et de l'érosion conséquente de ses frontières. Dans ce contexte, la politique de police dans le secteur de la Manche, avec le projet du tunnel sous la Manche, devenait fondamentale et nécessitait d'être repensée. La fondation du groupe TREVI en 1975, en réponse à l'activité terroriste en Europe qu'Interpol était réticente à traiter, amorça le processus qui fit basculer la question policière dans le domaine de la politique européenne. Mais ce furent des événements postérieurs qui le consolidèrent et le rendirent applicable à des délits ordinaires. En 1990, les délégués français à la CCIC firent un bilan de l'état des accords de liaison et firent des propositions d'avenir ${ }^{37}$. Le réseau policier local-transnational était solide. Le directeur de la Police de l'air et des frontières du Pas-de-Calais nota que son service avait un officier de liaison et que les visites des deux côtés de la Manche étaient régulières. Il souligna de même l'excellence des relations avec la Belgique et les Pays-Bas. Un cas pratique allait néanmoins se présenter bientôt : le tunnel sous la Manche. Les policiers français estimèrent qu'il serait souhaitable qu'il y eût une présence de la police française et britannique des deux côtés de la Manche. Il rapporta qu'il y avait eu de nombreuses réunions où, pour la première fois, la Gendarmerie nationale était représentée dans une structure policière transnationale " destinée à organiser au mieux la coopération et l'interpénétration future de nos services quand le tunnel serait ouvert à la circulation ". Mais il fallait répondre à d'autres besoins nécessaires, notamment " l'harmonisation des législations européennes en fonction des problèmes auxquels se trouvaient confrontés les services de police... ". La nomination par le directeur général de la Police nationale et le ministre de l'Intérieur d'un officier de liaison à Londres même fut un autre signal fort des choses à venir. Il fut suggéré que les officiers de liaison des différents services devraient être stationnés soit dans les locaux de la Direction générale de la Police nationale ou dans ceux de la P.A.F. à Paris. Les autres délégués exprimèrent alors la crainte que trop d'officiers opérant à des niveaux différents conduiraient à une fragmentation organisationnelle. Le délégué belge fit remarquer qu'Interpol avait déjà désigné 17 officiers européens de liaison; un officier néerlandais représentant le bureau d'Interpol de La Haye indiqua l'existence de la structure TREVI et des accords de Schengen. Cet officier pensait qu'en raison de la complexité des accords de transnationalisation policière en Europe, " la CCIC devrait 
rester une réunion de collègues et ne pourrait aller au-delà de certaines limites sans commettre d'erreurs ou sans perdre son rôle moteur ". L'un des représentants britanniques, arguant du fait qu'il serait impossible de multiplier les officiers de liaison en raison du nombre des spécialisations criminelles et juridiques, demeurait persuadé que " le contact direct entre policiers se connaissant parfaitement était plus profitable ". En 1991, d'autres sujets furent abordés et le chef du bureau britannique d'Interpol devait déclarer ${ }^{38}$ : " Il y a une zone grise, au regard de qui peut faire quoi en relation avec les enquêtes outre-mer; en même temps, Interpol, en tant qu'organisation, et le bureau londonien d'Interpol reconnaissent la qualité du travail effectué sous les auspices de la liaison transfrontalière de la Manche. Il faut aussi reconnaître qu'il existe un besoin de centralisation, d'un interface par lequel toutes les grandes enquêtes policières concernant le secteur devraient passer ou tout au moins être supervisées. En fait, le Royaume-Uni va ratifier très prochainement la Convention européenne d'assistance mutuelle [juridique] de 1957, et toutes les enquêtes de cette envergure devront désormais passer par l'autorité centrale désignée. Ce seront le Home Office ou, pour les cas urgents, le bureau d'Interpol. Ceci, naturellement n'empêchera pas la coopération locale de continuer sur une base transfrontalière ". Les débats sur la transmission des informations dans les polices des différents pays montrèrent que les anciennes barrières institutionnelles demeuraient. Les délégués de la PJ française rapportèrent " que des problèmes surgirent quand la Gendarmerie se vit confier des enquêtes internationales ". Ces problèmes chroniques de communication entre deux services d'un même pays signifiaient que la communication au niveau régional était jugée inadaptée et que toutes les recherches devaient passer par les bureaux centraux. En Belgique, les problèmes venaient du fait que les enquêtes devaient transiter par le biais des magistrats locaux pour aboutir au niveau national et ensuite être transmises aux autorités étrangères, les demandes venant de l'étranger suivant une procédure inverse. Cela avait posé de sérieux problèmes quand les douanes britanniques s'étaient adressées directement à la police locale. Quant à la police britannique, elle révéla qu'elle avait des difficultés dans la coordination de la liaison douanes-police, aux échelons local et régional, alors qu'elle était assez bonne aux niveaux supérieurs (la fondation du NCIS en Angleterre n'avait pas encore eu lieu). Ainsi, en dépit de plus d'une décennie de coopération réussie, la fragmentation administrative se faisait toujours sentir. Ces failles n'étaient pas comblées, or les gouvernements nationaux s'apprêtaient à redéfinir leur contrôle des opérations policières, en multipliant les infrastructures policières transnationales au niveau inter-gouvernemental (TREVI et plus tard Europol), ou en redéfinissant la place des autorités policières centrales et le rôle d'Interpol. Concernant ce dernier, les délégués des différents bureaux centraux nationaux d'Interpol investirent dans le développement technique des communications, tout particulièrement à propos de l'Automated Search Facility. Ce système technologique dernier cri fut programmé par Interpol dès 1984, lorsque le nouveau Président (un ancien membre des services secrets américains) inaugura l'époque de la prédominance anglo-américaine au sein de l'agence. Cela permit à Interpol de passer du code morse au courrier électronique pratiquement en une nuit ${ }^{39}$. Mais Interpol n'était pas le seul " courtier " en systèmes d'information. Existait aussi le projet du Schengen Information System, bien que le Royaume-Uni n'ait pas signé l'accord ; l'idée d'Europol était aussi dans l'air. Le souci principal, par rapport à tous ces systèmes de données, était d'assurer la précision des données et de respecter leur conformité à la règle de transmission de " renseignement strictement nécessaire ". 
Néanmoins, ces avancées technologiques globales sont postérieures aux systèmes de stockage de données et de communications dans la région de la Manche. Tandis que ces développements techniques semblaient promettre une croissance de l'entreprise policière transnationale, l'infrastructure organisationnelle au sein de laquelle la " révolution de l'information policière " se produisait, laissait présager un aboutissement logique moins certain. Les fondations sur lesquelles la politique transnationale policière européenne avait été construite étaient des systèmes policiers nationaux fragmentés. Or, quand l'entreprise policière dépassa le niveau des États-Nations, cette fragmentation ne s'allégea pas et l'existence de différents services de police transnationaux à des macro-niveaux (TREVI et Europol en sont au moins deux) contribua à la prolonger ${ }^{40}$. On peut établir un parallèle avec les structures de la future union monétaire. Par rapport à l'union monétaire, les technicités de la coopération politique ne seront pas simples. La Banque centrale européenne contrôlera la politique monétaire ; les ministres des finances auront leur mot à dire sur la politique des taux de change; les politiques fiscales (dans certaines limites) demeureront du ressort des gouvernements nationaux. Dans le cas de la coopération policière, les structures sont encore plus complexes. Si chaque pays n'a qu'une banque centrale, il existe toujours plusieurs polices (en dépit de quelques changements intervenus récemment dans certains pays). La police transnationale locale En 1991, la police du Kent réévalua ses capacités de liaison dans la partie anglaise de la Manche. Les relations avec leurs collègues de Calais, particulièrement la PAF et la PJ, étaient très bonnes. Les rapports, formalisés en 1989 au cours d'une série d'entretiens entre le Chief Constable du Kent et le préfet du Pas-de-Calais, étaient donc consolidés au plus haut niveau, ce qui permettait de les confirmer au niveau local. La sécurité publique, les opérations de secours ainsi que le contrôle aux frontières, le contrôle du crime, les télécommunications et les procédures opérationnelles furent les sujets abordés au cours des réunions de planification du tunnel sous la Manche. Ces débats furent répercutés au niveau opérationnel lors d'une série de réunions avec les personnels de police concernés. Simultanément, les développements au niveau macro-européen des politiques d'accords de coopération policière firent qu'on se rendit compte des conséquences importantes qu'elles auraient sur la politique transnationale dans ce secteur. La police du secteur de la Manche adhérait largement à l'opinion courante qui considérait que la marche vers le marché unique amplifierait le problème chronique du crime transfrontalier. Tout le monde s'accorda sur la nécessité d'une vaste mutation du travail policier transnational. Des adaptations organisationnelles permettant de répondre très vite à une situation fluide, mouvante, furent adoptées par le KC (Kent Constabulary). L'Unité européenne de liaison fut créée au sein du KC : les officiers de liaison du CID (Division des enquêtes criminelles) et ceux du Special Branch Ports Unit furent amalgamés en une seule unité opérationnelle qui, en assumant ces fonctions côte à côte avec la liaison combinée Europe/Interpol, unifiait le renseignement criminel. L'ELU créa une base de données informatique concernant toutes les enquêtes de police transfrontalières et, en coopération avec le bureau central de renseignements de la police du Kent (CIB), une base de données séparée concernant toutes les personnes qui avaient des liens avec le crime transfrontalier. Toutes les fonctions de liaison étaient rassemblées en un même lieu, simplifiant les tâches. L'ELU, basée à Folkstone, au terminal du tunnel sous la Manche, devint un centre-clé des communications dans tout le secteur ${ }^{41}$. Aujourd'hui, l'unité facilite les enquêtes et tout le travail policier afférent: de la localisation des objets perdus ou volés et des services 
de secours ou de traduction à la communication des renseignements dans les enquêtes transnationales de longue durée. Elle permet une large dissémination à l'étranger des informations sur les fichiers de criminels et de données informatisées de toute nature, entre réseaux locaux et régionaux, partout dans le Royaume-Uni, jusqu'aux fichiers de l'ordinateur national de la police (PNC) et le bureau des permis de conduire (DVLB). Elle sert aussi de canal pour l'information en provenance de l'étranger vers les contacts policiers du Royaume-Uni, y compris le NCIS. Des enquêtes, des recherches venant de Suisse, de Grèce, d'Espagne et de Gibraltar peuvent être gérées aussi facilement que celles en provenance d'Ostende, mais la majeure partie du travail effectué concerne la zone anglaise de la région de la Manche. La plupart des services de police opérant dans ce secteur, même ceux qui n'ont pas participé à la CCIC, pour l'essentiel les gendarmeries belge et française, sont intégrés au réseau. Les communications policières ont fait un bond qualitatif en passant à la communication électronique. À partir de 1990, l'ELU a coopéré au développement du site Linguanet, un système de courrier électronique qui, depuis 1996, couvre la totalité de la région qui va de Rotterdam (à l'Ouest) à Felixstowe dans l'Essex (au Nord), à Bruges, Lille et Boulogne (au Sud) et à Folkstone (sur la côte britannique de la Manche) ${ }^{42}$. La révolution de l'information a rendu l'information policière routinière, au point que le rôle de la CCIC est maintenant remis en cause. Ce n'est plus un forum d'échanges d'informations et de renseignements criminels. Les archives du début des années 1970 sont remplies de rapports détaillés d'enquêtes criminelles, les archives de l'année 1996 ne font état que d'un seul cas (celui d'un délinquant sexuel récidiviste qui a quitté l'Angleterre pour la Belgique). L'essentiel des débats s'est focalisé sur les innovations technologiques, comme, par exemple, la possibilité d'un système automatique de relevés des plaques minéralogiques, utilisable aux terminaux des ferries, et les compte rendus des réorganisations affectant les services de police membres de la CCIC, et sur l'éventualité de la constitution en termes de référence de la CCIC elle-même: d'une certaine manière, elle est aujourd'hui victime de son succès. Conclusion James Clifford a noté dans un récent ouvrage ${ }^{43}$ que le mouvement et le voyage sont des caractéristiques essentielles de l'être humain. Il y évoque l'image " du village traditionnel comme un séjour transitoire ", faisant remarquer combien il serait tentant de conclure qu'il n'y a pas de meilleure image de la post-modernité qu'un nouvel ordre de mobilité, d'histoires sans histoire ". Mais c'est précisément parce que l'être humain est historiquement en mouvement, qu'il s'éloigne de la post-modernité. Au contraire, le travail du voyage devient pour Clifford, l'élément crucial d'une modernité inachevée. Nous devons ici être prudent et éviter cette tendance des sciences sociales à vouloir dire les mêmes choses en usant d'un vocabulaire différent et en le déguisant en débat. Peu importe de quelle modernité nous parlons, ce qui est en jeu c'est la réalité que les termes tentent de décrire; en d'autres mots, c'est une question d'ontologie. L'enjeu est l'étendue de la transformation historique dans l'histoire contemporaine. En l'écrivant au présent, on peut découvrir soit une rupture substantielle avec le passé ou un continuum de développement. C'est ce que nous avons essayé de faire, dans le contexte limité du microcosme qu'est la Manche. Il parait évident que si les mouvements migratoires sont des caractéristiques propres à l'être humain, les formes techniques de leur contrôle ne le sont pas. Tout d'abord, on doit considérer que les formes de transport actuelles ont rendu les mouvements de masse beaucoup plus faciles à une échelle globale. L'échelle de la mobilité géographique a fait un saut quantitatif. Le mouvement de masse de l'industrie touristique en est l'un des indicateurs. Le 
phénomène souterrain du " tourisme de la drogue " ou du " tourisme du sexe " (en particulier celui lié à la pédophilie) était impensable auparavant, quand le voyage était l'apanage de quelques uns. Les mouvements massifs de population sont devenus une autre forme de confrontation. Ceux qu'on a qualifiés de " migrants économiques ", de " réfugiés politiques " ou d'" immigrants illégaux " sont devenus l'objet d'un débat d'intérêt général. Une tendance qui a existé auparavant, mais qui est une caractéristique de notre époque. Autrefois, les émigrants entreprenaient un long et périlleux voyage, avant de s'installer dans un pays d'accueil et d'être coupés de leur patrie d'origine. Aujourd'hui, ce voyage peut se faire en quelques heures, et le maintien du lien avec le pays d'origine se fait sans problèmes. Il suffit de se rappeler le mouvement migratoire d'Europe de l'Est vers les États-Unis à la fin du siècle dernier et de le comparer avec les mouvements actuels ${ }^{44}$. Alors que les moyens de transport ont renforcé la mobilité humaine, au-delà de tout ce qui était imaginable, les progrès technologiques en communication, en stockage d'informations, en capacité de recherche et d'analyse ont multiplié la capacité apparente de surveillance et de contrôle de ces mouvements. Un fait de ce type est observable dans le secteur de la Manche. Le stockage de données informatisées et de gestion de grandes quantités d'informations a atteint une capacité énorme. Le SIS (Schengen Information System) peut conserver en mémoire et analyser quinze millions de dossiers, des estimations courantes prévoyant une gestion de deux millions et demi d'enquêtes d'ici à la fin de la décennie. Dans une époque où la préoccupation essentielle est l'insécurité porteuse de dislocation sociale, ces systèmes d'information gigantesques sont devenus le symbole, s'ils n'en sont pas les instruments, du rempart contre le désordre social. Les autres moyens de surveillance le sont aussi : on peut considérer sous cet angle, l'usage des écrans de surveillance destinés à assurer la sécurité des terminaux du tunnel sous la Manche. Avant même son ouverture, il fut révélé à une conférence de la CCIC que 120 écrans surveilleraient les 26 points d'accès du terminal, à Coquelles, du côté français, contrôlés par 20 moniteurs situés dans la salle de contrôle de la PAF. Cet oeil électronique, s'il peut dissuader, voire empêcher certaines formes de crimes à l'intérieur des sites, fournit surtout un symbole d'ordre très fort, destiné aux populations qui empruntent ces " corridors de sécurité "45. Pourtant, il demeure un doute sur la capacité instrumentale de ces technologies à pouvoir offrir la sécurité promise, ce, pour deux raisons: le schéma organisationnel qui circonscrit les opérations de police dans la Manche, et par extension partout ailleurs, est fragmenté. Cela crée des frictions organisationnelles qui ne sont que partiellement surmontées par le sens de la mission et les efforts des personnels de police engagés. Ainsi, de temps en temps, les policiers montent des opérations qui réussissent, des criminels sont arrêtés, mais cela renforce surtout le sentiment d'insécurité en mettant en évidence l'étendue du problème. De plus, à côté de la fragmentation bureaucratique, il y a le manque d'harmonisation des lois criminelles. Depuis 1968, l'européanisation de la juridiction criminelle a énormément avancé ${ }^{46}$, bien plus que prévu, mais nous nous trouvons toujours en présence d'une vision " à distance " du système européen de justice criminelle. Elle n'est seulement qu'un futur possible. La Convention de Schengen (que le Royaume-Uni n'a toujours pas ratifié) n'est pas une garantie d'homogénéité, car tant que son interprétation sera de la responsabilité des juges nationaux il n'y a aucune garantie que ses dispositions seront harmonisées ${ }^{47}$. Comme nous l'avons démontré dans cet article, ce manque d'uniformisation peut tourner à l'avantage de la police, mais il peut être un frein à une coopération policière réussie. Tous ces faits nous amènent à 
tirer une conclusion pessimiste: la technologie offerte par les nouveaux moyens de surveillance, d'analyse et d'échange d'informations demeure plus symbolique que pratique. Il est clair que tout système de police transnational doit plus aux efforts du personnel qui lui donne vie qu'à la rationalité de la bureaucratie et l'étroitesse du cadre juridique qui le circonscrivent. Les questions d'efficience mises à part, il est nécessaire d'aborder un thème, lié à ce que nous avons évoqué précédemment, sans l'avoir abordé : les Droits de l'Homme et la justice naturelle. Peuvent-ils s'intégrer dans un système policier qui, en raison de sa complexité organisationnelle et par nature, est opaque ? Derrière ces développements, apparaît, en contrepoint, un renversement historique qui peut se caractériser comme la tendance lourde dirigeant les sentiments d'insécurité des peuples qui ont la sensation d'être agressés. En simultanéité avec les développements techniques que nous avons décrits, et en partie conduits par eux, de profonds changements affectent l'État-Nation. Une évaluation des processus qui contribuent à cette restructuration globale des systèmes de gouvernance dépasse le cadre de cet article. Mais cette mutation profonde du mode de gouvernance en Europe depuis le milieu du siècle, qui s'est accélérée dans la période de l'après-Guerre Froide, est évidente. Ce mouvement vers un " système d'État transnational " implique la délégation de responsabilités de gouvernement au niveau régional même si des institutions transnationales usurpent ou, au moins, tentent de s'approprier des pouvoirs appartenant à l'origine à l'État-Nation. Ceci est une évidence en ce qui concerne les systèmes policiers. Les systèmes policiers nationaux se réajustent en fonction des besoins du nouvel ordre mondial, les systèmes policiers transnationaux se développent très vite. Or, l'aperçu que nous avons donné du système de police dans la Manche montre qu'il est un patchwork complexe plutôt qu'une couverture de sécurité. La fragmentation demeure la caractéristique du système transnational étatique dans la nouvelle Europe. Jusqu'à une date récente, des modes stato-centrés de contrôle social permettaient la gestion des suspects habituels: terroristes, trafiquants de drogue, trafiquants de main d'oeuvre immigrée, mafieux et autres délinquants. Aujourd'hui, les agences transnationales revendiquent aussi le droit de les gérer. C'est une entreprise coûteuse, surtout du fait de sa nature fragmentée. Ironie de l'histoire, elle est l'expression d'une gouvernementalité qui semble flottante (sans gouvernail) et qui n'est pas débitable d'une source identifiable de pouvoir politique légitimé. On doit pourtant souligner que, en dépit de la nature fragmentée de cette coopération policière, le patchwork qui émerge de son activité a sa propre cohérence. Ces changements frappants dans la manière de faire la police dans le monde qui nous entoure nous amènent à nous poser la question sur la " crise actuelle " et le tournant que nous avons atteint, dépassé ou se profilant devant nous. La vision que l'on peut en avoir est contradictoire : on voit à l'oeuvre à la fois la fragmentation et l'unité dans la capacité technologique à gérer le désordre. Le maintien de l'ordre est soumis à la contingence, au chaos du destin et du hasard. En effet, les policiers et les experts du contrôle social ne sont pas différents de nous - dans un monde mouvant, ils ont perdu, tout comme nous d'ailleurs, le sens de la fixité institutionnelle et sociale alors que nous devons déjà assumer une nouvelle identité et une nouvelle cohérence. Si nous considérons qu'une cohérence nouvelle et distincte peut émerger au siècle prochain, nous pouvons dire, pour l'instant, que nous sommes simplement post-modernes. 


\section{NOTES}

1. James W. E. Sheptycki est Research Fellow à l'ESRC (Grant Number H524 2700061 94). Cette recherche a bénéficié du soutien de la Nuttfield Foundation, de la British Academy, du Lindsay Bequest Fund, du Wilson Memorial Fund, et de l'Université d'Edinburgh.

2. Je dois remercier ici pour leur concours, le Detective Inspector D. F. Gallagher de la police du Kent et le commissaire J. Denolf de la Police judiciaire de Bruges qui m'ont facilité l'accès aux Archives de la CCIC. Ces archives (en trois langues, anglais, français et néerlandais) sont conservées à Bruges.

3. CCIC, Maidstone, 15 mai 1968.

4. CCIC, Brighton, 23 avril 1970.

5. CCIC, Bruges, 29 avril 1970.

6. R. Reiner, Chief Constables, Oxford, Clarendon Press, 1991.

7. Plusieurs unités de police ayant compétence au niveau national ont précédé la création du NCIS ; les principales furent le National Drugs Intelligence Unit et le Footbal Intelligence Unit. Ces unités n'avaient pour mission que de renseigner. Aujourd'hui encore, seule la Special Branch a le pouvoir d'arrêter un suspect partout dans le Royaume-Uni.

8. Les premiers sont habituellement d'anciens officiers de police, appointés par le HMI pour inspecter les services de police et s'assurer de leur qualification et de leur efficacité. Les seconds sont des fonctionnaires civils chargés de vérifier si la politique du gouvernement est appliquée.

9. Voir M. Wintle, " Policing the Liberal State in the Netherlands ", Policing and Society, 1996, vol. 6, pp. 181-197, et R. Doornebal, The Police of Netherlands, The Hague, Ministry of the Interior, 1994.

10. T. Jones, Policing and Democracy in the Netherlands, London, PSI, 1995 ; M. Punch, Policing the Inner City, London, Macmillan, 1979.

11. Il est intéressant de signaler que les juges néerlandais qui sont responsables de la conduite des enquêtes ne participèrent jamais aux réunions de la CCIC, au contraire de leurs collègues belges qui ont des pouvoirs similaires.

12. T. Jones, op. cit. ; James W. E. Sheptycki, " The Global Cops Cometh ", British Journal of Sociology, à paraître (1997) et L. de Witte, " The Belgian Perpective on the Internationalization of Police Cooperation " in C. Fijnaut (ed.), Internationalisation of Police Co-operation in Western Europe, The Hague, Deventer, Kluwer, 1993.

13. P. de Cock, The Belgian Police and European Police Co-operation, University of Edinburgh, Department of Politics, Working Paper Series, 1992.

14. Seule exception à la règle, un représentant de la Gendarmerie belge assista à la réunion de Winchester les 6 et 7 mai 1975, réunion spéciale consacrée au trafic de stupéfiants. La police judiciaire belge protesta en affirmant qu'elle était la seule agence policière qualifiée au niveau international et que la Gendarmerie devait être exclue de toute action internationale. Une motion adoptée à l'unanimité fut votée déclarant " qu'il était du ressort des délégués nationaux d'accepter la présence ou non d'observateurs d'une autre agence de police, fait qui serait signifié par écrit à la Gendarmerie " (CCIC, Winchester, 6-7 mai 1975). Onze ans plus tard, la question refit surface et les mêmes responsables policiers s'opposèrent à l'intégration de la 
Gendarmerie belge, qui n'a jamais fait partie de la CCIC (CCIC, Rotterdam, 14-15 mai 1986).

15. J. Roach and J. Thomaneck, The Police and Public Order in Europe, London, Croom Helm, 1985.

16. P. J. Stead, The Police of France, New-York, Macmillan, 1983.

17. M. Anderson, " The French Police and Co-operation in Western Europe " in C. Fijnaut, The Internationalisation of Police Co-operation in Europe, Deventer, Kluwer, 1993.

18. C. Horton, Policing Policy in France, London, PSI, 1995.

19. M. Anderson, Policing the World, Oxford, Clarendon Press, 1989.

20. CCIC, Lille, 16 avril 1969.

21. CCIC, Bruges, 29 Avril 1970.

22. CCIC, Chelmsford, 7 avril 1971.

23. CCIC Lille, op. cit.

24. Le Morse est resté le principal moyen de codage des communications d'Interpol jusqu'aux années 1980. Comme l'expliqua le secrétaire général André Bossard, " le morse ne coûtait rien ", même s'il reconnaissait que ce n'était pas un mode de communication défendable. (F. Bresler, Interpol, London, Madarin, 1992). Interpol Londres n'abandonna le télex qu'au début des années 1990. Nos propres observations sur le terrain nous permettent de confirmer que le personnel des communications utilise encore le Morse. Au cours des trois premières phases de la transnationalisation, Interpol était technologiquement en retard.

25. CCIC, Rotterdam, 20-21 septembre 1973.

26. CCIC, Pas-de-Calais, 23-24 mai 1978.

27. CCIC, Maidstone, 10-11 mai 1977. Le réseau transnational dans la zone de la Manche a eu à traiter des centaines d'infractions de toute nature. Ce cas a été choisi en raison du nombre de problèmes qu'il posait du point de vue de la police des frontières.

28. CCIC, Pas-de-Calais, op. cit.

29. CCIC, Chelsmford, 15-16 mai 1979.

30. CCIC, Chantilly, 28-30 mai 1984 ; Rotterdam, 14-15 mai 1986.

31. CCIC, Chelsmford, 15-16 mai 1979 ; CCIC, Pas-de-Calais, 23-24 mai 1978.

32. CCIC, Chantilly, 28-30 mai 1984 ; CCIC,14-15 mai 1986, Rotterdam.

33. CCIC Chantilly, op. cit.

34. Voir James W. E. Sheptycki, " Law Enforcement, Justice and Democracy in the Transnational Arena ", International Journal of the Sociology of Law, 1996, vol. 24, pp. 61-75, pour une discussion du renforcement de la jurisprudence transnationale.

35. M. Anderson, The Internationalisation ..., op. cit., p. 26.

36. CCIC, Westcliffe-on-sea, 16-18 mai 1989.

37. CCIC, Rouen, 15-17 mai 1990.

38. CCIC, Sussex, 14-16 mai 1991.

39. PRSU, Bulletin 42, Home Office, Science and Technology Group, London, Police Requirements Support Unit, 1992 ; F. Bresler, op. cit.

40. James W. E. Sheptycki, " Transnational Policing and the Makings of a Postmodern State ", British Journal of Criminology, 1995, vol. 35, n 4, pp. 613-635.

41. Voir les tableaux 1 et 2 en annexe.

42. Un système similaire, le projet Palma (Police, Aachen, Leige et Maastricht) existe dans la région du Limbourg et est certainement appelé à se développer. Palma et 
Linguanet utilisant les mêmes protocoles de transfert des données, il sera possible de les connecter entre eux.

43. J. Clifford, Routes, Travel and Translation in the Late Twentieth Century, Cambridge, Harvard University Press, 1997.

44. Un bon exemple du profond bouleversement perceptible est celui du Khat, cette préparation de feuilles de Catha Edulis, consommée couramment en Afrique de l'Est et dans la Péninsule arabique. Cette drogue, consommée par les communautés Somalies d'East London, Liverpool, Sheffield et Middleborough, n'est pas une substance prohibée et la National Drugs Intelligence Unit estimait, en 1990, à 4000 le nombre de ses utilisateurs à Londres (tous Somaliens, Arabes ou immigrants de la première génération). La plante devant être consommée fraîche, son importation se fait sur une base quotidienne.

45. James W.E. Sheptycki, " Policing, Postmondernity and Transnationalisation ", British Journal of Criminology, à paraître (1997).

46. C. Harding, P. Fennell, P. Jörg, B. Swart, Criminal Justice in Europe, Oxford, Clarendon Press,1995 ; J. W. E. Sheptycki, " Review Article on Criminal Justice in Europe ", Howard Journal, 1996, vol. 36, n 1, pp. 109-112.

47. C. Joubert et H.Bevers, Schengen Investigated, The Hague, Kluwer, 1995 ; J. W. E. Sheptycki, " Review of Schengen Investigated ", The International Journal of Evidence and Proof, à paraître (1997).

INDEX

Index géographique : Royaume Uni

Mots-clés : coopération internationale, police 